\title{
Search for QCD Instanton-Induced Processes in DIS at HERA
}

\author{
Stanislav Mikocki ${ }^{* \dagger}$ \\ IFJ PAN, Cracow, Poland \\ E-mail: mikocki@ifj.edu.pl
}

\begin{abstract}
Signals of QCD instanton-induced processes are searched for in neutral current deep-inelastic scattering at the electron-proton collider HERA in the kinematic region defined by the Bjorkenscaling variable $x>10^{-3}$, the inelasticity $0.2<y<0.7$ and the photon virtuality $150<Q^{2}<$ $15000 \mathrm{GeV}^{2}$. The search is performed using $\mathrm{H} 1$ data corresponding to an integrated luminosity of $351 \mathrm{pb}^{-1}$. No evidence for the production of QCD instanton-induced events is observed. Upper limits on the cross section for instanton-induced processes between $1.5 \mathrm{pb}$ and $6 \mathrm{pb}$, at 95\% confidence level, are obtained depending on the kinematic domain in which instantons could be produced. Compared to earlier publications, the limits are improved by an order of magnitude and for the first time are challenging predictions.
\end{abstract}

XXIV International Workshop on Deep-Inelastic Scattering and Related Subjects

11-15 April, 2016

DESY Hamburg, Germany

\footnotetext{
*Speaker.

$\dagger$ On behalf of the H1 Collaboration.
} 


\section{Introduction}

The Standard Model of particle physics contains certain anomalous processes induced by instantons which violate the conservation of baryon and lepton number $(B+L)$ in the case of electroweak interactions and chirality in the case of strong interactions $[1,2]$. In quantum chromodynamics (QCD), the theory of strong interactions, instantons are non-perturbative fluctuations of the gluon field. They can be interpreted as tunnelling transitions between topologically different vacua.

The theory and phenomenology for the production of instanton-induced processes at HERA in neutral current (NC) electron/positron-proton collisions has been worked out by Ringwald and Schrempp $[3,4]$. Instanton processes predominantly occur in photon gluon $(\gamma g)$ fusion processes as sketched in figure 1. The partonic final state contains pair of quark and anti-quark of each light flavour and some number of gluons, where one of the quarks (anti-quarks) acts as the current quark $\left(q^{\prime \prime}\right)$. The partons from the instanton subprocess are distributed isotropically in the instanton rest frame $\left(\overrightarrow{q^{\prime}}+\vec{g}=0\right)$. The hadronic final state is expected to have following signature: the densely populated band in the pseudo-rapidity $(\eta)$ with the particles of relatively high transverse momentum isotropically distributed in azimuth, a high particle multiplicity and a current jet emerging from the outgoing current quark $q^{\prime \prime}$.

The instanton production cross section at HERA, $\sigma_{\text {HERA }}^{(I)}$, is determined by the cross section of the instanton subprocess $q^{\prime}+g \stackrel{(I, \bar{I})}{\rightarrow} X$. which is calculable in instanton perturbation theory. It involves the distributions of the size $\rho$ of instantons and of the distance $R$ between them. There is a one-to-one relation between the variables in momentum space $Q^{\prime 2}$ and $x^{\prime}$ and the space variable $\rho$ and $R$. Large $Q^{\prime}$ and $x^{\prime}$ values correspond to small $\rho$ and large $R / \rho$, respectively. The region of validity of the instanton perturbative calculation was derived by confronting this calculation with non-perturbative lattice simulations of the QCD vacuum [5]. The obtained limits $\rho \lesssim 0.35 \mathrm{fm}$ and $R / \rho \gtrsim 1.05$ can be translated into regions of the kinematical variables $Q^{\prime 2} \geq Q_{\min }^{\prime 2} \simeq\left(30.8 \times \Lambda_{\overline{M S}}^{n_{f}}\right)^{2}$ and $x^{\prime} \geq x_{\min }^{\prime} \simeq 0.35$ [6] where $\Lambda_{\overline{M S}}^{n_{f}}$ is the QCD scale for $n_{f}$ flavours. The cross section depends significantly on the strong coupling $\alpha_{s}$, or more precisely on $\Lambda_{\overline{M S}}^{n_{f}}$.

The analysis is performed in the kinematic region defined by $0.2<y<0.7$ and $150<Q^{2}<$ $15000 \mathrm{GeV}^{2}$. In this kinematic region, and additionally requiring $Q^{\prime 2}>113 \mathrm{GeV}^{2}$ and $x^{\prime}>0.35$, the cross section predicted by QCDINS Monte Carlo generator [6] is $\sigma_{\text {HERA }}^{(I)}=10 \pm 3 \mathrm{pb}$, using the QCD scale $\Lambda \frac{(3)}{M S}=339 \pm 17 \mathrm{MeV}$ [7]. The quoted uncertainty of the instanton cross section $\sigma_{\text {HERA }}^{(I)}$ is obtained by varying the QCD scale by one standard deviation.

\section{Data Selection, Definition of the Observables and the Search Strategy}

This analysis [8] is performed using the full $e^{ \pm} p$ collision data set taken in the years 20032007 by the $\mathrm{H} 1$ experiment corresponding to a total integrated luminosity of $351 \mathrm{pb}^{-1}$ The High $Q^{2}$ neutral current DIS events are selected to cover the phase space region defined by $0.2<y<0.7$, $x>10^{-3}$ and $150<Q^{2}<15000 \mathrm{GeV}^{2}$. The data sample consists of about 350000 events.

The observables used to discriminate the instanton-induced contribution from that of the standard DIS processes are based on the hadronic final state (HFS).

All HFS objects are boosted to the hadronic centre-of-mass frame (HCM). Jets are defined by the inclusive $k_{T}$ algorithm as implemented in FastJet, with the massless $P_{T}$ recombination scheme 
Jets are required to have transverse energy in the $\mathrm{HCM}$ frame $E_{T, \text { jet }}>3 \mathrm{GeV}$. Additional requirements on the transverse energy and pseudorapidity of the jets in the laboratory frame are imposed in order to ensure that jets are contained within the acceptance of the Liquid Argon calorimeter and are well calibrated. The events are selected by requiring at least one jet with $E_{T, \text { jet }}>4 \mathrm{GeV}$. The jet with the highest transverse energy is used to estimate the 4-momentum $q^{\prime \prime}$ of the current quark (see figure 1). $Q^{\prime 2}$ is reconstructed from the particles associated with the current jet and the photon 4-momentum.

The hadronic final state objects belonging to the current jet are not used in the definition of the following observables. A "instanton band" ( $I$-band) in pseudo-rapidity with a width of \pm 1.1 units in $\eta$ is defined around the mean $\bar{\eta}$. The number of charged particles in the $I$-band $n_{B}$ is measured.

An approximate instanton rest frame, where all hadronic final state objects in the $I$-band are distributed isotropically, is defined by $\overrightarrow{q^{\prime}}+\xi \vec{P}=0$ where $\xi=0.076$ is a constant determined from studies of the instanton simulation. The axes $\vec{i}_{\min }$ and $\vec{i}_{\max }$ are found for which in the instanton rest system the summed projections of the 3-momenta of all hadronic final state objects in the $I$-band are minimal or maximal. The relative difference between $E_{\mathrm{in}}=\sum_{h}\left|\vec{p}_{h} \cdot \vec{i}_{\max }\right|$ and $E_{\text {out }}=\sum_{h}\left|\vec{p}_{h} \cdot \vec{i}_{\min }\right|$ is called $\Delta_{B}=\left(E_{\text {in }}-E_{\text {out }}\right) / E_{\text {in }}$. This quantity is a measure of the transverse energy weighted azimuthal isotropy of an event. For isotropic events $\Delta_{B}$ is small while for pencil-like events $\Delta_{B}$ is close to unity. Using two methods to calculate the invariant mass of the quark gluon system, $W_{I}, x^{\prime}$ is reconstructed as $x_{\mathrm{rec}}^{\prime}=\left(x_{1}^{\prime}+x_{2}^{\prime}\right) / 2$, where $x_{i}^{\prime}=Q_{\mathrm{rec}}^{\prime 2} /\left(W_{I, i}^{2}+Q_{\mathrm{rec}}^{\prime 2}\right)$ with $W_{I, 1}^{2}=\left(q_{\mathrm{rec}}^{\prime}+\xi P\right)^{2}$ and $W_{I, 2}^{2}=\left(\sum_{h} p_{h}\right)^{2}$ where the sum runs over the HFS objects in the $I$-band.

The measured distributions of the five observables $E_{T, \text { jet }}, n_{B}, x_{\mathrm{rec}}^{\prime}, \Delta_{B}$ and $E_{\text {in }}$ are compared in figure 2 (upper left) to the expectations from the standard DIS QCD models (RAPGAP [9], DJANGOH [10]) and from the instanton model (QCDINS). The data are reasonably well described by the reweighted standard DIS Monte Carlo simulations.

\section{Search for Instanton-Induced Events and Results}

A multivariate discrimination technique is employed to increase the sensitivity to instanton processes. The PDERS method as implemented in the TMVA ROOT package [11] is used.

The set of observables, $E_{T, \text { jet }}, n_{B}, x^{\prime}, \Delta_{B}$ and $E_{\text {in }}$, has been chosen since it provides the best signal to background separation. and the distributions of these variables are overall well described by both Monte Carlo simulations. The distribution of the discriminator $D$ is shown in figure 2 (upper right). Taking into account the systematic uncertainties, the discriminator distribution is described by the DIS Monte Carlo simulations in the background dominated region (low D values). The instanton signal peaks at large values of the discriminator.

A signal region is defined for $D>D_{\text {cut }}=0.86$, optimised for a determination of the instanton signal from event counting. The distributions of the expected instanton signal and of the background are shown in figure 2 (lower right). No excess of events is observed and the DJANGOH MC describes the data well, while the prediction of RAPGAP is systematically above the data.

No evidence for QCD instanton-induced processes is observed and the data are used to set exclusion limits.

The upper limit is determined from a $\mathrm{CL}_{s}$ statistical analysis using the method of fractional event counting [12], optimised for the presence of systematic uncertainties, which are treated as 
Gaussian distributions. Limits are calculated using the full range of the disciminator distribution as shown in figure 2 (upper right).

Figure 2 (lower left) shows the behaviour of the observed $\mathrm{CL}_{s}$ as a function of the instanton signal cross section. In this study the total instanton cross section is taken as a free parameter, whereas the signal shape is taken from the QCDINS simulation. The observed limit is $2 \mathrm{pb}$ at $95 \%$ $\mathrm{CL}$ and the predicted cross section $10 \mathrm{pb}$ estimated using QCDINS is excluded by the H1 data.

In order to assess the sensitivity of the instanton cross section on the kinematic variables $x_{\min }^{\prime}$ and $Q_{\text {min }}^{\prime 2}$, limits are also determined as a function of the lower bounds $x_{\min }^{\prime}$ and $Q_{\min }^{\prime 2}$. The results are shown in figure 3 (left), where the observed confidence levels, using the QCDINS predictions, are shown in the $\left(x_{\min }^{\prime}, Q_{\min }^{\prime 2}\right)$ plane. A less model-dependent search is presented in figure 3 (right). Here, limits on the instanton cross section are determined as a function of the parameters $x_{\min }^{\prime}$ and $Q_{\text {min }}^{\prime 2}$, using the signal shapes predicted by QCDINS. No uncertainty on the instanton cross section normalisation is included in this determination of the experimental cross section limit. The most stringent exclusion limits of order $1.5 \mathrm{pb}$ are observed for large $Q_{\min }^{2}$ and small $x_{\min }^{\prime}$. For increasing $x_{\min }^{\prime}$ the limits are getting weaker. At the nominal QCDINS setting, $x_{\min }^{\prime}=0.35$ and $Q_{\text {min }}^{\prime 2}=113 \mathrm{GeV}^{2}$, one expects to find back an exclusion limit of $2 \mathrm{pb}$, as discussed with figure 2 (lower left). The limit in figure 3 (right), however, is observed to be somewhat better, because the theory uncertainty on the cross section normalisation is included in figure 2 (lower left) but not in figure 3 (right).

\section{Conclusions}

A search for QCD instanton-induced processes is presented in neutral current deep-inelastic scattering at HERA in the kinematic region : $x>10^{-3}, 0.2<y<0.7$ and $150<Q^{2}<15000 \mathrm{GeV}^{2}$. The search is performed using $\mathrm{H} 1$ data corresponding to an integrated luminosity of $351 \mathrm{pb}^{-1}$.

Several observables of the hadronic final state of the selected events are exploited to identify a potentially instanton-enriched sample. Two Monte Carlo models, RAPGAP and DJANGOH, are used to estimate the background from the standard NC DIS processes. The instanton-induced processes are modelled by the program QCDINS. In order to extract the expected instanton signal a multivariate data analysis technique is used. No evidence for QCD instanton-induced processes is observed. In the kinematic region defined by the theory cut-off parameters $x_{\min }^{\prime}=0.35$ and $Q_{\text {min }}^{\prime 2}=113 \mathrm{GeV}^{2}$ an upper limit of $2 \mathrm{pb}$ on the instanton cross section at $95 \% \mathrm{CL}$ is determined Thus, the corresponding predicted instanton cross section of $10 \pm 3 \mathrm{pb}$ is excluded by the $\mathrm{H} 1$ data. Limits are also set in the kinematic plane defined by $x_{\min }^{\prime}$ and $Q_{\min }^{\prime 2}$. These limits may be used to assess the compatibility of theoretical assumptions such as the dilute gas approximation and theoretical instanton properties such as their size and distance distributions, with the H1 data.

Compared to earlier publications, QCD instanton exclusion limits are improved by an order of magnitude and are challenging predictions based on instanton perturbative calculations with parameters derived from lattice QCD data.

\section{Acknowledgements}

We thank A. Ringwald and F. Schrempp for many helpful discussions. We are grateful to the HERA machine group whose outstanding efforts have made this experiment possible. We also 


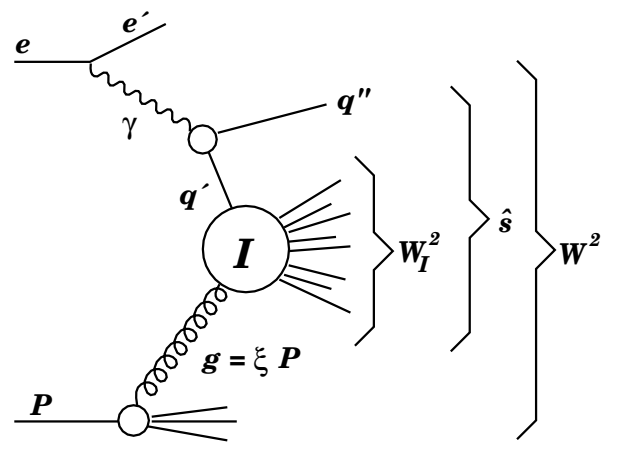

NC DIS variables:

$$
\begin{array}{lc}
\mathbf{s}=(e+P)^{2} & Q^{2}=-\gamma^{2}=-\left(e-e^{\prime}\right)^{2} \\
\mathbf{x}=Q^{2} /(2 P \cdot \gamma) & \mathbf{y}=Q^{2} /(s x) \\
W^{2}=(\gamma+P)^{2}= & Q^{2}(1-x) / x \\
\hat{\mathbf{s}}=(\gamma+g)^{2} & \xi=x\left(1+\hat{s} / Q^{2}\right)
\end{array}
$$

Variables of the instanton subprocess:

$$
\begin{aligned}
& Q^{\prime 2} \equiv-q^{\prime 2}=-\left(\gamma-q^{\prime \prime}\right)^{2} \\
& \mathbf{x}^{\prime} \equiv Q^{\prime 2} /\left(2 g \cdot q^{\prime}\right) \\
& W_{I}^{2} \equiv\left(q^{\prime}+g\right)^{2}=Q^{\prime 2}\left(1-x^{\prime}\right) / x^{\prime}
\end{aligned}
$$

Figure 1: Sketch of an instanton-induced preocess and kinematic variables.
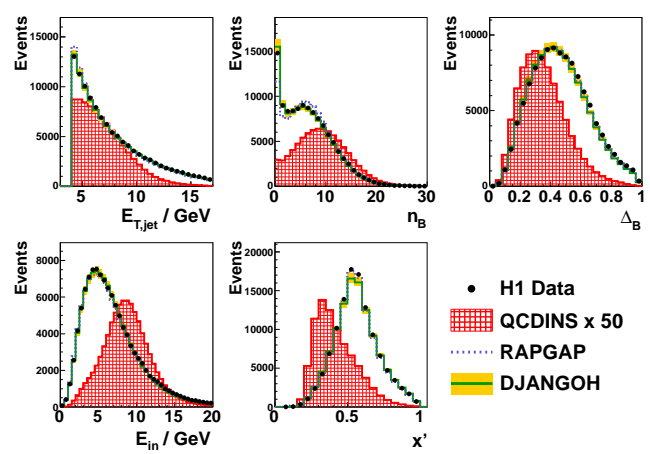

QCDINS x 50

RAPGAP

DJANGOH
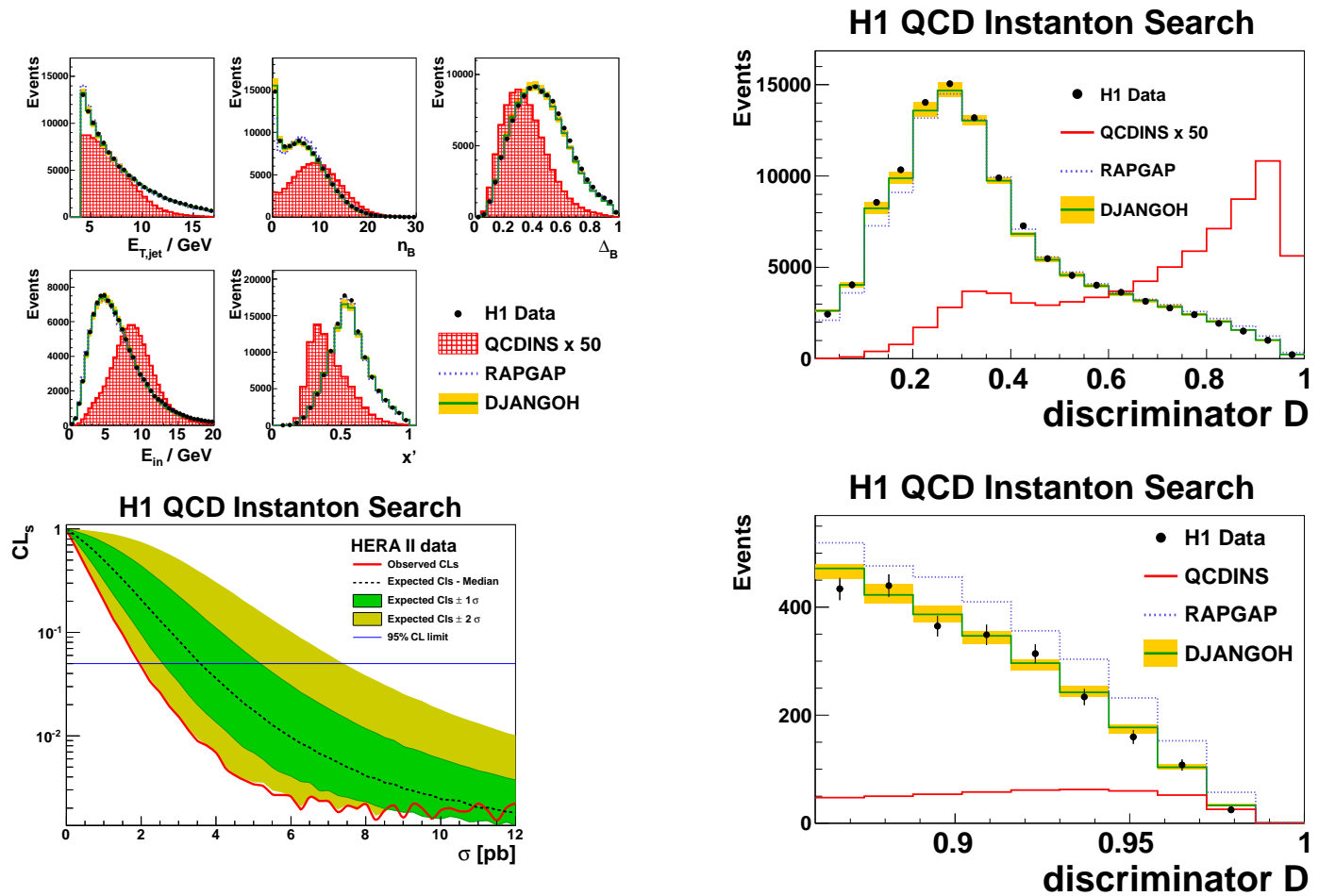

Figure 2: Upper left: Distributions of the observables used in the multivariate analysis. Upper right: The distribution of the discriminator $D$. Lower left: Observed $\mathrm{CL}_{s}$ as a function of the instanton cross section. Lower right: The distribution of the discriminator $D$ in signal region $D>0.86$.

thank the DESY directorate and our funding agencies for support. We would like to give credit to all partners contributing to the EGI computing infrastructure for their support.

\section{References}

[1] A. A. Belavin, A. M. Polyakov, A. S. Shvarts and Y. S. Tyupkin, Pseudoparticle Solutions Of The Yang-Mills Equations, Phys. Lett. B 59 (1975) 85.

[2] G. 't Hooft, Symmetry Breaking Through Bell-Jackiw Anomalies, Phys. Rev. Lett. 37 (1976) 8; G. 't 

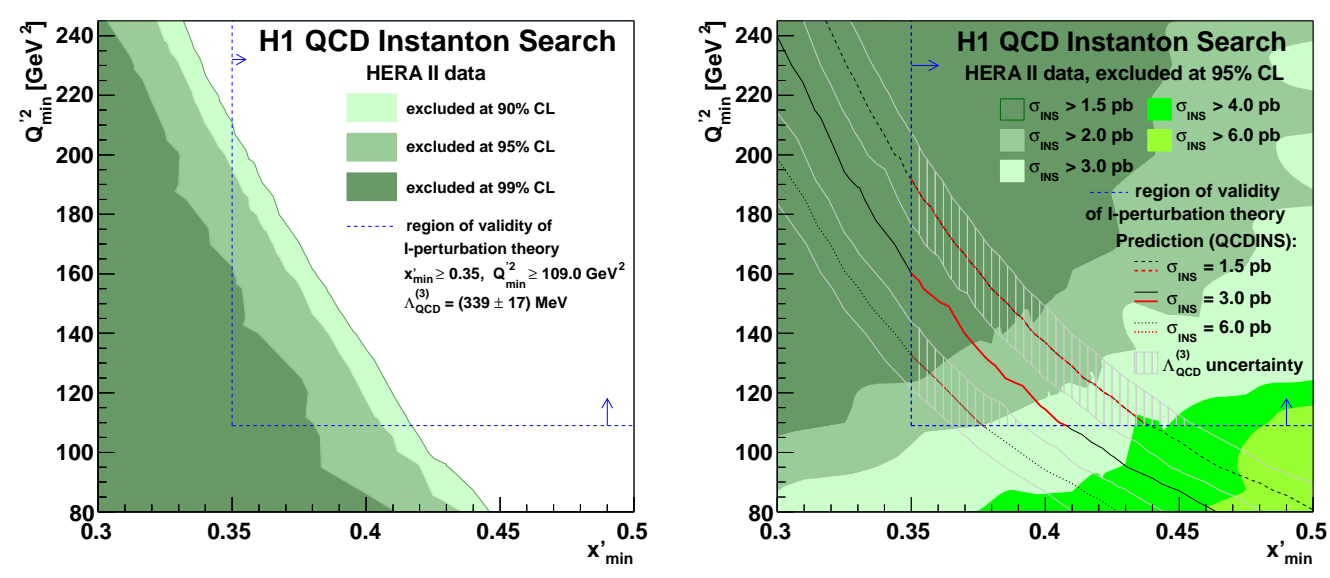

Figure 3: Left: Instanton production exclusion limits as a function of $x_{\min }^{\prime}$ and $Q_{\min }^{\prime 2}$. Right: Upper limits on the instanton cross section at $95 \%$ confidence level, as a function of $x_{\min }^{\prime}$ and $Q_{\min }^{\prime 2}$.

Hooft, Computation Of The Quantum Effects Due To A Four-Dimensional Pseudoparticle, Phys. Rev. D 14 (1976) 3432; [Erratum-ibid. D 18 (1976) 2199].

[3] A. Ringwald and F. Schrempp, Towards the Phenomenology of QCD-Instanton Induced Particle Production at HERA, Quarks '94 (Vladimir, Russia, 1994), D. Grigoriev et al., Eds., Proc. of the 8th Int. Seminar, pp. 170, [hep-ph/9411217].

[4] A. Ringwald and F. Schrempp, Instanton-induced cross sections in deep-inelastic scattering, Phys. Lett. B 438 (1998) 217 [hep-ph/9806528].

[5] A. Ringwald and F. Schrempp, Confronting instanton perturbation theory with QCD lattice results, Phys. Lett. B 459 (1999) 249 [hep-lat/9903039].

[6] A. Ringwald and F. Schrempp, QCDINS 2.0: A Monte Carlo generator for instanton-induced processes in deep-inelastic scattering, Comput. Phys. Commun. 132 (2000) 267 [hep-ph/9911516]. Available at http://www.desy.de/ t00fri/qcdins/qcdins.html.

[7] S. Aoki et al., Review of lattice results concerning low-energy particle physics, Eur. Phys. J. C 74 (2014) 2890 [arXiv:1310.8555].

[8] V. Andreev et al. [H1 Collaboration], Search for QCD instanton-induced processes at HERA in the high- $Q^{2}$ domain, Eur. Phys. J. C 76 (2016) no.7, 381 doi:10.1140/epjc/s10052-016-4194-6 [arXiv:1603.05567 [hep-ex]].

[9] H. Jung, Hard diffractive scattering in high-energy e p collisions and the Monte Carlo generator RAPGAP, Comput. Phys. Commun. 86 (1995) 147.

[10] K. Charchula, G. A. Schuler and H. Spiesberger, title Combined QED and QCD radiative effects in deep inelastic lepton - proton scattering: The Monte Carlo generator DJANGO6, Comput. Phys. Commun. 81 (1994) 381.

[11] A. Höcker et al., TMVA - Toolkit for Multivariate Data Analysis, PoS ACAT (2007) 040 [physics/0703039].

[12] P. Bock, Computation of confidence levels for exclusion or discovery of a signal with the method of fractional event counting, JHEP 0701 (2007) 080 [hep-ex/0405072]. 